\title{
Enlightenment, Revolution and the periodical press. Edited by Hans-Jürgen Lüsebrink and Jeremy D. Popkin
}

\section{Peter Balazs}

\section{(2) OpenEdition}

Journals

Édition électronique

URL : http://journals.openedition.org/studifrancesi/30286

DOI : 10.4000/studifrancesi.30286

ISSN : 2421-5856

Éditeur

Rosenberg \& Sellier

\section{Édition imprimée}

Date de publication : 1 avril 2006

Pagination : 155-156

ISSN : 0039-2944

\section{Référence électronique}

Peter Balazs, «Enlightenment, Revolution and the periodical press. Edited by Hans-Jürgen Lüsebrink and Jeremy D. Popkin », Studi Francesi [En ligne], 148 (XLX | I) | 2006, mis en ligne le 30 novembre 2015, consulté le 21 avril 2021. URL : http://journals.openedition.org/studifrancesi/30286 ; DOI : https:// doi.org/10.4000/studifrancesi.30286

Ce document a été généré automatiquement le 21 avril 2021.

\section{cc) (†)}

Studi Francesi è distribuita con Licenza Creative Commons Attribuzione - Non commerciale - Non opere derivate 4.0 Internazionale. 


\title{
Enlightenment, Revolution and the periodical press. Edited by Hans- Jürgen Lüsebrink and Jeremy D. Popkin
}

\author{
Peter Balazs
}

\section{RÉFÉRENCE}

Enlightenment, Revolution and the periodical press. Edited by HANS-JÜRGEN LÜSEBRINK and JEREMY D. POPKIN, Oxford, Voltaire Foundation, 2004 (SVEC 2004:6), pp. 226.

1 Depuis quelques années les recherches poursuivies dans le domaine de l'histoire des périodiques ont révélé le rôle extrêmement important que les feuilles, les journaux et les revues ont joué au siècle des Lumières. Les deux éditeurs du présent volume ont sélectionné des articles qui étudient l'implication de la presse périodique dans la politique et dans la culture des pays européens au siècle des Lumières et dans l'ère révolutionnaire.

2 L'introduction de JEREMY D. POPKIN et de JACK CENSER (Some paradoxes of the eighteenthcentury periodical, pp. 3-21) fournit une vue d'ensemble des caractéristiques de la presse du XVIII ${ }^{e}$ siècle. Popkin reprend l'analyse de Kaspar Stieler (auteur de Zeitungs Lust und Nutz, 1965) concernant la raison d'être du nouveau médium: la presse permet aux hommes de satisfaire leur inclination naturelle à se renseigner sur le monde dans lequel ils vivent et cette curiosité, loin d'être condamnable, doit servir de base à une sorte d'éducation morale à laquelle les journalistes doivent soumettre leurs lecteurs. Censer, quant à lui, se concentre sur l'analyse des éléments de continuité et de rupture entre la presse de l'Ancien Régime et celle de la Révolution.

HANS-JÜRGEN LÜSEBRINK (Horizons médiatiques et ouvertures interculturelles dans la presse au dix-huitième siècle), pp. 22-32) apporte à ses lecteurs, tout en anticipant sur les résultats 
des articles qui figurent dans le volume, quelques observations précieuses sur les rapports et les interférences entre le champ journalistique et le champ littéraire. Ces interférences se manifestent à plusieurs niveaux: le premier, l'importance du littéraire dans le corpus des périodiques est indiscutable. Le deuxième niveau est celui des registres rhétoriques, tandis que le troisième renvoie à des homologies structurelles entre genres journalistiques et genres littéraires. Lüsebrink observe également que la presse du XVIII ${ }^{e}$ siècle reste perméable aux lettres et correspondances manuscrites, ainsi qu'aux formes de communication orale. Le dernier aspect de la presse du XVIII siècle que Lüsebrink met en relief est son intercultura1ité, qui renvoie finalement à la problématique générale des transferts culturels.

Dans son article consacré aux «nouvelles à la main» (News and newsletters in Portugal [1703-1754], pp. 35-45) retrouvées dans les archives portugaises,JOAO LUIS LISBOA montre que ce genre particulier - qui a joué en France, comme en Angleterre un rôle très important dans la propagation des nouvelles politiques et militaires - est présent au Portugal depuis le début du siècle des Lumières au moins. Les feuilles manuscrites à parution quasi régulière (dont Lisboa fournit une bibliographie détaillée en annexe) ont dans un certain sens complété les gazettes imprimées dans la mesure où les premières ont pu rendre compte des événements que les dernières devaient taire du fait de la censure.

5 L'étude de MARIE-CHRISTINE SKUNCKE (Press and political culture in Sweden at the end of the Age of liberty, pp. 81-102) traite des périodiques qui paraissent en Suède dans les années 1760. Les deux partis principaux du royaume (aristocrates et royalistes, ennemis implacables) prennent conscience très tôt que la presse périodique constitue une arme potentiellement très efficace dans leur course au pouvoir. Quoique très engagés et partiaux, les journaux suédois qui rendent régulièrement compte des événements du Riksdag poursuivent une véritable éducation civique des lecteurs. La victoire des royalistes met bientôt fin à la courte période caractérisée par la liberté de la presse, néanmoins les années 1766-1772 ont indiscutablement marqué la société suédoise.

6 L'article bien documenté de ANNE MARIE MERCIER-FAIVRE (Une lecture fantasmatique de la Gazette d'Amsterdam au temps des Lettres Persanes [1720-1721]: le cas du despotisme oriental, pp. 46-80) montre l'abîme qui sépare la représentation de l'Orient qu'on trouve sur les pages de la Gazette d'Amsterdam des années 1720-1721 de celle qui caractérise les textes littéraires et romanesques composés â la même époque. Alors que les auteurs littéraires insistent volontiers sur la figure mythique du despote oriental et tentent de séduire leurs lecteurs avec des romans de sérail, la gazette en question s'approche de la personne de Mehmet Effendi, plénipotentiaire persan avec l'intention déclarée de donner une description minutieuse et détaillée de ses gestes. Mercier-Faivre montre que l'objet 'Orient' qui naît des ces bribes journalistiques livrées semaine après semaine n'est pas moins fantasmé et romanesque que celui qui se dessine par exemple dans les Lettres persanes. Quoique depuis le XVII ${ }^{\mathrm{e}}$ siècle textes littéraires et presse périodique tendent progressivement à diverger, il est indiscutable que les deux types de textes sont nourris par les mêmes 'représentations' ou images préfabriquées.

7 La contribution de BERNADETTE FORT (Le discours politique dans les Salons des Mémoires secrets, pp. 102-113) montre comment certains éléments du discours politique se manifestent dans un texte à vocation culturelle, notamment dans les critiques d'art des Mémoires secrets, attribués depuis deux siècles à Louis Petit de Bachaumont. Le critique d'art présent aux Salons - les expositions bisannuelles de l'Académie Royale - ne se 
contente pas de soumettre les représentations de la personne du roi, des grands de sa cour et de ses ministres à un jugement d'ordre purement esthétique; au contraire, ses remarques et ses allusions portent souvent sur des questions politiques extrêmement délicates. L'auteur de ces critiques particulières attire l'attention de ses lecteurs sur la grande vulnérabilité de la monarchie française, cette dernière ayant miné sa propre autorité par une alternance d'autoritarisme et de timidité.

8 L'étude de MARTIN STUBER (Journal and letter: the interaction between two communications media in the correspondance of Albrecht von Haller, pp. 114-141), consacrée à l'analyse de l'impressionnante correspondance de l'érudit suisse Albrecht von Haller et à sa collaboration au périodique Gottingische Gelehrte Anzeigen montre que, contrairement à ce qu'on pourrait croire, l'ascendance d'un nouveau médium (la presse) n'a point exterminé l'ancien (les lettres et la communication manuscrite): les renseignements obtenus par l'intermédiaire de ses correspondants européens (1200, selon le calcul de Stuber) permettent à Haller d'alimenter sa revue. L'article, extrêmement bien documenté, montre que les textes imprimés dans les périodiques transmettent à leurs lecteurs l'idéal de la République des Lettres, composée d'égaux, tous à la recherche de la vérité; quant â la correspondance personnelle de Haller, elle nous révèle les passions, les ambitions et les jalousies des particuliers impliqués dans les réseaux de sociabilité savante de l'époque.

Dans son article (The Spectator of the Spectators: Jacques-Vincent Delacroix, pp. 145-157) consacré prioritairement à la carrière de Jacques-Vincent Delacroix, M. L. G. PALLAREBURKR énumère les traits caractéristiques des imitations du Spectator, si nombreuses dans l'Europe du XVIII ${ }^{e}$ siècle. La revue immortelle d'Addison et de Steele devint un véritable exemple canonique, de sorte que certains des imitateurs se disputaient le titre du plus fidèle des disciples des deux maîtres britanniques. Delacroix est indiscutablement parmi les imitateurs les plus assidus: entre 1767 et 1830, il publie 15 ouvrages (périodiques et livres confondus) qui réfèrent, par leur titre, à la revue anglaise. Le journaliste français s'efforce tout au long de sa vie de définir en quoi consiste exactement d'être "spectateur», c'est-à-dire observateur impartial, capable d'exercer une véritable «magistrature morale» sur ses compatriotes dans les conditions historiques les plus diverses.

L'article d'ERIC NEGREL (Le journaliste-orateur: rhétorique et politique sans-culottes dans Le Publiciste de la République Française de Jacques Roux, juillet-octobre 1793, pp. 158-177) sur le personnage du journaliste-orateur analyse les articles publiés dans le Publiciste de la République Française dont les 29 numéros paraissent au cours de l'été 1793. Son rédacteur, Jacques Roux, représentant illustre des «Enragés» se voit comme celui qui reprend la plume tombée de la main de Marat, assassiné. Négrel analyse les formes rhétoriques à l'oeuvre dans cette revue qui dénonce «les royalistes, les fédéralistes, les égoïstes, les modérés, les accapareurs, les monopoleurs, les agioteurs, les intrigants, les traitres et les sangsues du peuple». Les appels au peuple, la dénonciation des ennemis, la justification de son patriotisme, le style oratoire et l'invocation d'un discours de vérité proviennent indiscutablement des pamphlets et de la communication orale; Jacques Roux ne fait que les transposer dans sa production journalistique afin de créer un «espace rhétorique» susceptible de soutenir les actions politiques des leaders Enragés. En agissant ainsi, Roux contribue à l'instauration d'un espace public démocratique, mais fondamentalement antiparlementaire dont le «peuple» est l'acteur principal, voire unique. 
11 SUSANNE LACHENICHT étudie (La presse des immigrants allemands en Alsace [1791-1799], pp. 178-198) les périodiques des immigrants allemands en Alsace, entre 1791 et 1797 . A partir de 1791, les deux départements alsaciens accueillent des milliers de réfugiés allemands, fascinés par la Révolution et chassés de leur pays d'origine par le durcissement de la politique des cours princières allemandes. Dans les années en question, ces «jacobins allemands» publient dix-huit journaux qui circulent non seulement dans la région alsacienne, mais également en Allemagne et en Suisse. Après avoir présenté au lecteur les méthodes de recherche mises en œuvre (analyse quantitative des sujets traités, analyse sémantique des articles, biographie des éditeurs, etc.), Lachenicht souligne que dans la perspective de l'histoire des idées, les journalistes alsaciens n'ont pas avancé d'opinions novatrices ou inattendues. L'intérêt véritable de son étude consiste en une analyse exacte de leur caractère éclectique, c'est-à-dire de la proportion du mélange des conceptions des différents partis politiques.

L'étude de PHILIPPE HARLING (The perils of 'French Philosophy': Enlightenment and Revolution in Tory Journalism, 1800-1832, pp. 179-197), consacrée à l'analyse du discours utilisé dans la presse Tory des trois premières décennies du XIX ${ }^{\mathrm{e}}$ siècle, montre que les conflits idéologiques de l'ère révolutionnaire ne cessent d'inquiéter les conservateurs du pays le plus stable du continent européen. Les périodiques proches du parti Tory (de la Quarterly Review à l'Anti-Jacobin Review Harling en énumère une demi-douzaine, chacune possédant une griffe particulière) dénoncent les dangers inhérents à la propagation outre-Manche de la philosophie athéistique française, égoïste et malsaine, capable de miner toute autorité politique et morale. On ne sait pas si le ton parfois apocalyptique des articles étudiés par Harling reflètent ou inspirent l'angoisse des lecteurs anglais, mais le mérite principal de l'analyse est indiscutablement de montrer que le torysme d'après-guerre ne se caractérisait pas uniquement par ce pragmatisme confiant que les historiens lui attribuent volontiers. 